\title{
Evaluation of MR elastography as a response parameter for transarterial chemoembolization of colorectal liver metastases
}

\author{
Thomas J. Vogl ${ }^{1}$ • Simon S. Martin ${ }^{1,2} \cdot$ Addison A. Johnson $^{2} \cdot$ Yannick Haas $^{1}$
}

Received: 25 September 2019 / Revised: 16 January 2020 / Accepted: 3 February 2020 / Published online: 21 February 2020

(C) The Author(s) 2020

\begin{abstract}
Objective The aim of this study was to evaluate magnetic resonance elastography (MRE) as a response parameter in patients who received transarterial chemoembolization (TACE) for the treatment of colorectal liver metastases.

Materials and methods Forty-two patients (29 male patients; mean age, 61.5 years; range, 41-84 years) with repeated TACE therapy of colorectal liver metastases underwent on average 2 repetitive magnetic resonance imaging (MRI) and MRE exams in 4- to 6-week intervals using a 1.5-T scanner. MRE-based liver stiffness measurements were performed in normal liver parenchyma and in metastatic lesions. Moreover, the size of the liver metastases was assessed during treatment and compared with the results of the MRE analysis.

Results Liver metastases showed a significantly higher degree of stiffness compared with the normal liver parenchyma $(p<0.001)$. However, only a weak correlation was found between the lesion size and stiffness $(r=-0.32, p=0.1)$. MRE analysis revealed an increase in stiffness of the colorectal liver metastases from 4.4 to $7.1 \mathrm{kPa}$ after three cycles of TACE $(p<0.001)$. Also, the mean size of the metastases decreased from 17.0 to $11.3 \mathrm{~cm}^{2}(p<0.001)$. Finally, the entire liver stiffness increased from 2.9 to $3.1 \mathrm{kPa}$ over the three cycles of TACE therapy.

Conclusion In conclusion, MRE showed a significant change in stiffness and size of liver metastases. Therefore, MRE may provide an added value for an evaluation of treatment response in patients with colorectal liver metastases undergoing TACE.

Key Points

- MRE showed an increase in stiffness of the colorectal liver metastases during TACE therapy.

- Liver metastases showed a significantly higher degree of stiffness compared with the normal liver parenchyma.

- However, only a weak correlation was found between the lesion size and stiffness.
\end{abstract}

Keywords Liver $\cdot$ Chemotherapy $\cdot$ Metastasis $\cdot$ Therapeutic chemoembolization $\cdot$ Magnetic resonance imaging

\section{Abbreviations}

CRLM Colorectal liver metastases

CT Computed tomography

HCC Hepatocellular carcinoma

$\mathrm{kPa} \quad$ Kilo pascal

MRE Magnetic resonance elastography

MRI Magnetic resonance imaging

Thomas J. Vogl

t.vogl@em.uni-frankfurt.de

1 Department of Diagnostic and Interventional Radiology, University Hospital Frankfurt, Frankfurt, Germany

2 Department of Radiology and Radiological Science, Medical University of South Carolina, Charleston, SC, USA
RFA Radiofrequency ablation

TACE Transarterial chemoembolization

\section{Introduction}

Colorectal cancer is one of the most prevalent forms of cancer in the world [1], and the liver represents the most common site of metastases [2]. Overall, $50 \%$ of patients with colorectal carcinoma develop liver metastases and $20 \%$ of them have synchronous metastases, which generally show poor outcomes [3-6]. Partial liver resection can only be considered in patients with good liver function to avoid potential liver decompensation [7-9]. Systemic chemotherapy also has limited use for patients with unresectable liver metastases [8, 10-12]. Therefore, alternative therapies like RFA (radiofrequency 
ablation) and MWA (microwave ablation) are recommended for those patients [13-16].

If neither surgery nor local-ablative methods show response, transarterial chemoembolization (TACE) is an option for local tumor control. This therapy has shown promising results in patients with unresectable colorectal liver metastases [13]. TACE is based on the administration of a cytostatic substance directly into the tumor feeding vessel and shows fewer side effects compared with systematic chemotherapy. Due to the combination of direct chemotherapeutic application and vascular occlusion technique, the cytostatic substances are in contact with the targeted malignant cells for a longer period of time $[17,18]$. Magnetic resonance imaging (MRI) and computed tomography (CT) are frequently used for therapy assessment and follow-up. Magnetic resonance elastography (MRE) is a novel technique to be considered and can be applied to most patients undergoing MRI [19, 20]. MRE showed a higher accuracy and a better reproducibility compared with sonography-based elastographic measurements [21].

The aim of our study was to assess MRE as a response parameter in patients with TACE of colorectal liver metastases.

\section{Material and methods}

\section{Study design and population}

This retrospective study was approved by the institutional ethical committee of our university hospital. Data was collected between April 2017 and December 2017. All patients had met our inclusion criteria as follows: (a) age between 18 and 90 years old, (b) histopathological and/or radiological evidence of colorectal liver metastases, (c) current therapy with TACE, (d) no contraindications for MRI scanning. Exclusion criteria were as follows: (a) liver metastases of different origin, (b) inflammation in or around the liver, (c) abbreviations from the standard imaging protocol.

Forty-two patients (29 men and 13 women; mean age, 61.5 years; range, $41-84$ years) who had met the general inclusion and exclusion criteria were analyzed in the present study. The patients underwent regular treatment with TACE (mitomycin, gemcitabine, and lipiodol) and MRI with additional MRE. The MRI was performed either on the same day as the TACE therapy or in the following 2 days after the intervention, so that a range of 0-2 days in between MRE and TACE can be given. As treatment effects will vary over time and inconsistencies in timing between MRE snapshots and TACE could bias the results, patients with longer ranges were not included. The majority of MRI and MRE examinations $(93 \%)$ were performed immediately after TACE on the same day. All study patients underwent TACE in 4-6 weeks intervals. Twelve patients had evidence of metastases in the left liver lobe, while the other 30 showed metastases in the right lobe. In the case of multiple metastatic lesions in a single patient, only the largest lesion was evaluated. On average, 3.3 (range, 0-23) TACE interventions were performed before the first MRE imaging.

\section{TACE interventions}

After the puncture of a femoral artery, a pigtail catheter was inserted via the Seldinger technique [22]. Thereafter, a cobra or sidewinder catheter was placed in the celiac and superior mesenteric artery to visualize the vessels and liver metastases. In addition, the information from the MRI was used to identify the segmental, tumor-feeding artery using micro-catheters (size, 2.3-3.0 F) to prevent vasospasms. After positioning the catheters, the cytostatic substances were administered (mitomycin C $\left(8 \mathrm{mg} / \mathrm{m}^{2}\right.$ body surface), gemcitabine (500 $\mathrm{mg} / \mathrm{m}^{2}$ body surface), and cisplatin $\left(30 \mathrm{mg} / \mathrm{m}^{2}\right.$ body surface) in all patients. After administration of the chemotherapeutic substances, a selective injection of lipiodol was performed. The lipiodol capitation was not correlated with the efficacy of the TACE because of the frequently inhomogeneous appearance. All TACE therapies were performed using a robot-supported angiography system (Artis pheno, Siemens Healthineers), and the median time of the whole procedure was $35 \mathrm{~min}$ including the installation of the coil and positioning checks [22].

\section{MRI and MRE acquisition}

Unenhanced and contrast-enhanced MRI was performed directly before or after the intervention using gadobutrol (Gadovist $1 \mathrm{mmol} / \mathrm{ml}$, Bayer Healthcare). T1- and T2weighted MRI and MRE scans were acquired in transverse and sagittal orientation with $5 \mathrm{~mm}$ slice thickness using a 1.5-T system (MAGNETOM Avanto, Siemens). Commercially available system was used for MRE measurements (Resoundant) which consisted of an active driver located outside the scan room connected to passive actuators in the scan room. The time of vibrations was between $15 \mathrm{~s}$ for five slices of EPI (WIP measurement) and $23 \mathrm{~s}$ for a single slice of GRE. The median time for the MRE measurement including patient and hardware preparation was $18 \mathrm{~min}$. The applied sequence protocol consisted of the listed parameters in Table 1. All images were acquired during inspiratory breath hold and with a vibration frequency of $60 \mathrm{~Hz}$. The system evaluated magnitude image, phase image, wave image, color-coded elastogram, and confidence map. The stiffness metric was read in the magnitude of the complex shear modulus which could be read directly from the scanner outputs. 
Table 1 MRE protocol

\begin{tabular}{lc}
\hline MR parameter & Value \\
\hline Repetition time (TR) & $50 \mathrm{~ms}$ \\
Echo time (TE) & $21.1 \mathrm{~ms}$ \\
Bandwidth & $250 \mathrm{~Hz} /$ pixel \\
Field of view (FOV) & $400 \times 400$ \\
Matrix & $128 \times 128$ \\
NEX & 1 \\
Slice thickness & $10 \mathrm{~mm}$ \\
\hline
\end{tabular}

\section{MRE measurements}

The MRI data was matched with the MRE measurements using a dedicated software (MapIt Software, Siemens). This approach allows for a high-resolution segregation of intrahepatic structures and exact measurements of intrahepatic elastography, differentiating metastases and healthy tissue. Three different groups of patients with MRE imaging were investigated, as not every patient had the same number of performed MRE series due to the retrospective design of the study. Patients with one MRE examination $(n=16)$ were only used for the collection of the general elastography data. Patients with two $(n=11)$ or more $(n=15)$ MRE series were evaluated to assess therapy response (Fig. 1). A third data was calculated for patients with only two measurements via linear extrapolation, as more than one cycle is necessary for this evaluation. For this study, the MRI series were matched with MRE and merged to a tumor-stiffness 3D fusion image (Fig. 2). Evaluation of the treatment response included the following parameters: total extent of the liver $\left(\mathrm{cm}^{2}\right)$, total liver stiffness $(\mathrm{kPa})$ (including healthy parenchyma and tumor),
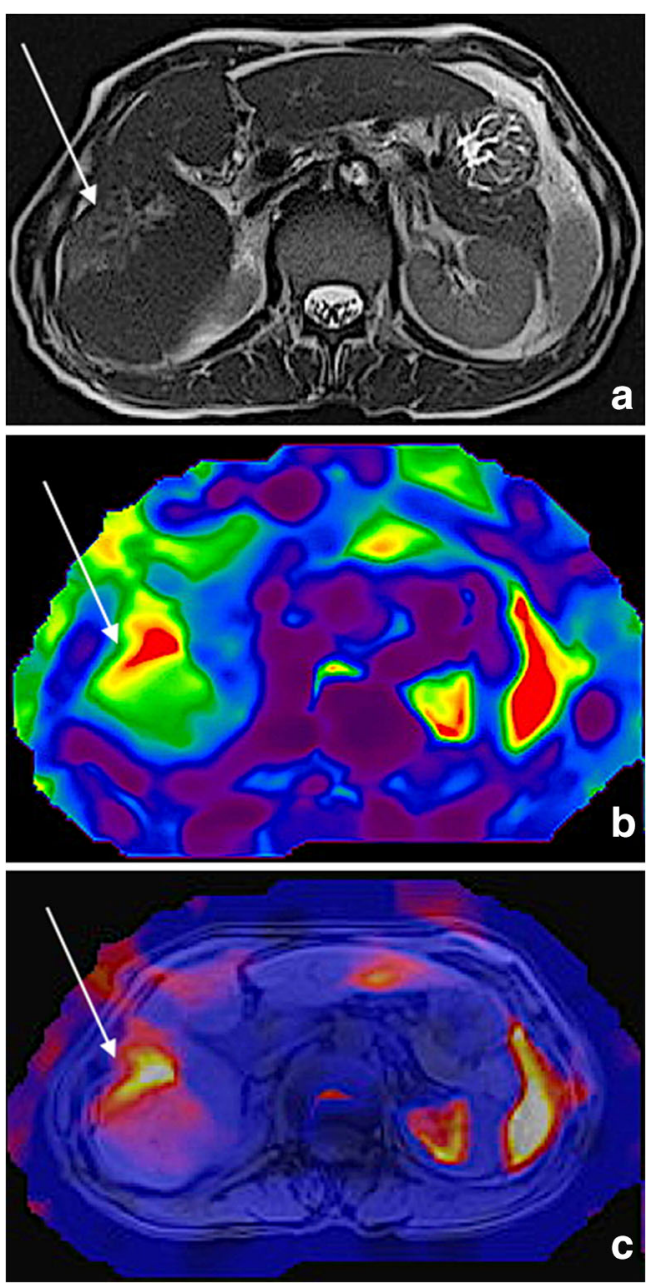

Fig. 2 A 64-old-male patient with colorectal liver metastasis in the right hepatic lobe. The metastasis is visualized in both images (arrows). The native MRI series (a) were matched with MRE data and merged to a tumor-stiffness 3D fusion for parameter analysis $(\mathbf{b}, \mathbf{c})$
Fig. 1 Flowchart of the study population. There were three groups of patients. Patients with one MRE imaging $(n=16)$ were only used for general

elastography data collection. The patients with two $(n=11)$ or more $(n=15)$ MRE series were further compared for size and stiffness evaluation during therapy

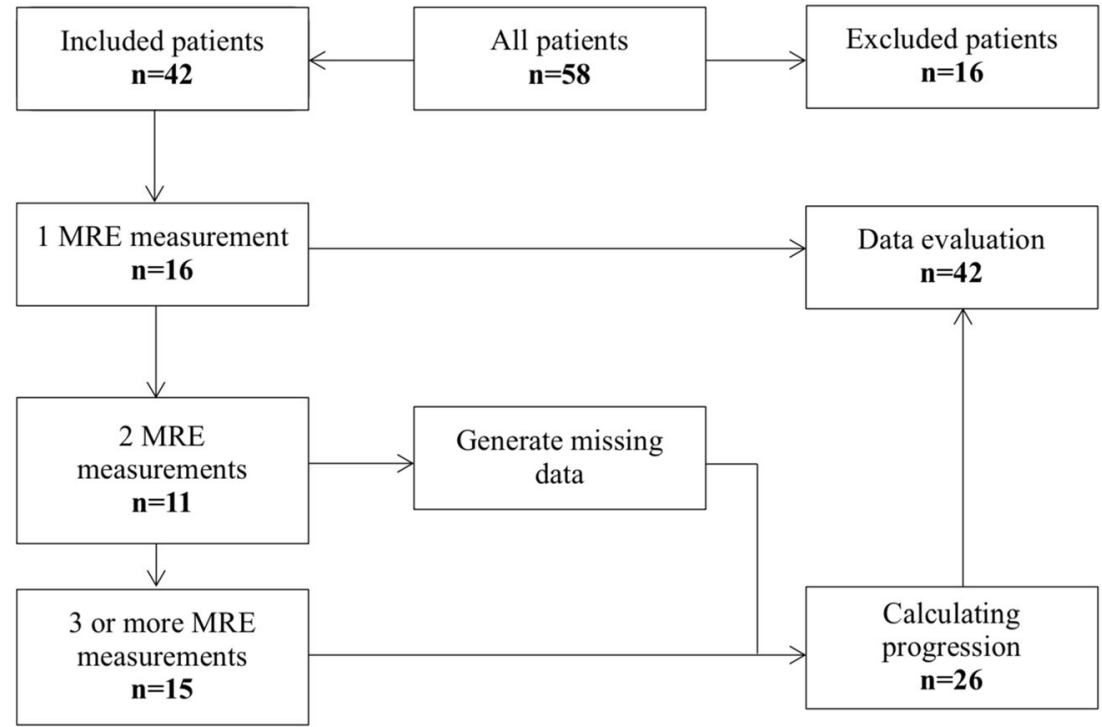


Fig. 3 A 64-old-male patient with colorectal liver metastasis in the right hepatic lobe (arrow) and TACE therapy. Evaluation of the treatment response included the following parameters: total liver extent $\left(\mathrm{cm}^{2}\right)$, total liver stiffness $(\mathrm{kPa})$, total liver MAP T1 (ms) and MAP T2 (ms), left and right lobe separated extent, stiffness and MAP T1/T2 measurement, metastasis extent, stiffness and MAP T1/T2. The images show the T1 image (a), as well as MRE measurements of the surrounding area (b with $4.7 \mathrm{kPa}$, $\mathbf{c}$ with $5.7 \mathrm{kPa}$ ) and the metastasis itself (d with $8.0 \mathrm{kPa}$ )
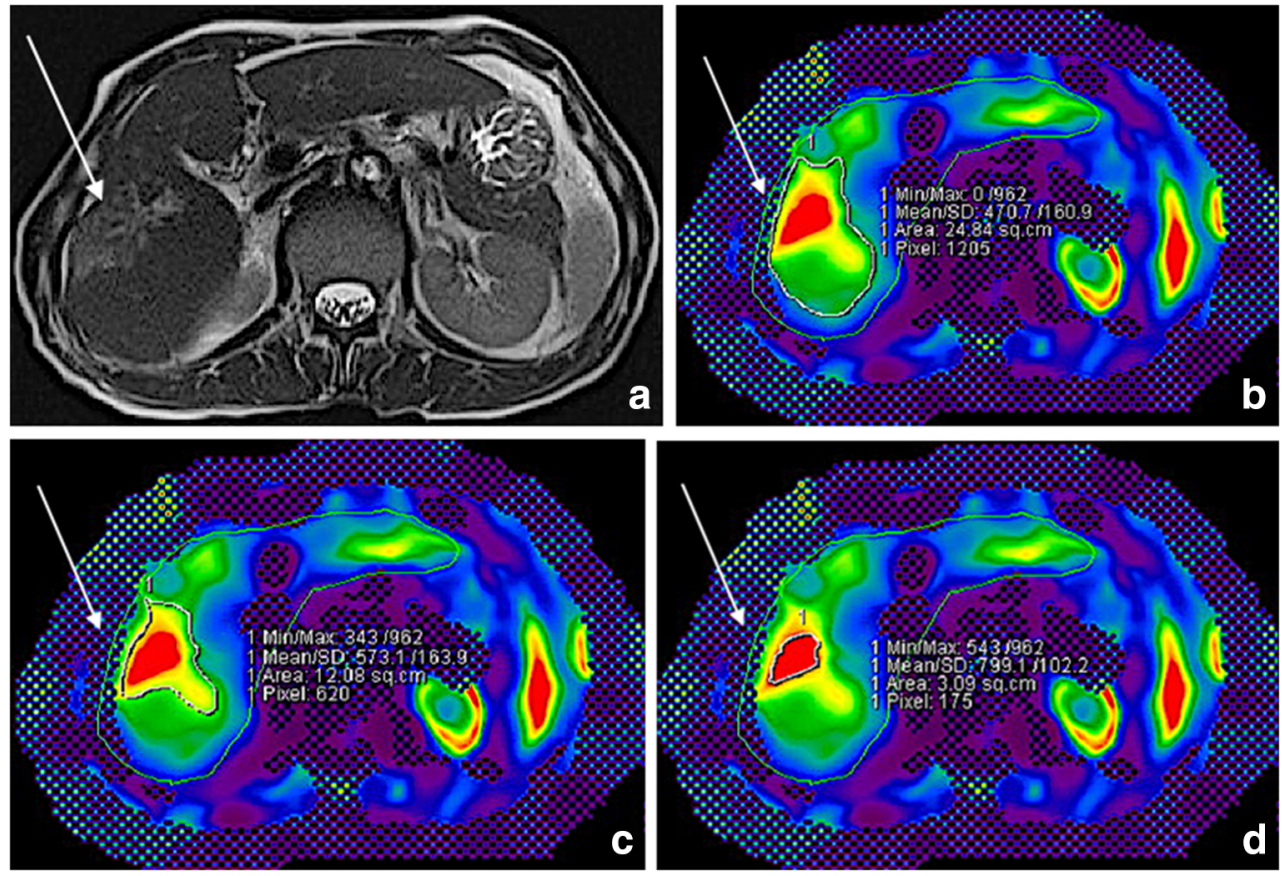

total liver MAP T1 (ms) and MAP T2 (ms), left and right lobe separated extent, stiffness and MAP T1/T2 measurement, metastasis extent, stiffness and MAP T1/T2 (Fig. 3). Additionally, measurements in the healthy liver tissue were performed for comparison.

\section{Statistical analysis}

Minimum and maximum values with standard deviations were calculated for continuous variables, whereas medians with ranges were calculated for categorical variables. The Kolmogorov-Smirnov test was applied to test for normal distribution. The Friedman test was used to determine whether differences between the measurements were significant. Statistical software (IBM SPSS Statistics, version 25, IBM) was used and a $p$ value $<0.05$ indicated statistical significance. Spearman's test was used to assess correlation.

\section{Results}

The mean size of the metastases decreased from 15.9 \pm $11.3 \mathrm{~cm}^{2}$ in the first MRI to $12.5 \pm 8.0 \mathrm{~cm}^{2}$ in the second
MRI and to $11.5 \pm 10.0 \mathrm{~cm}^{2}$ in the third MRI $(p=0.03)$. Simultaneously, a significant change in stiffness was detected in the liver metastases increasing from $4.8 \pm 1.4 \mathrm{kPa}$ in the first MRI to $7.0 \pm 2.6 \mathrm{kPa}$ in the third MRI $(p<0.001)$. In addition, the metastases $(5.9 \pm 2.2 \mathrm{kPa})$ were significantly stiffer than the normal liver parenchyma $(3.2 \pm 1.0 \mathrm{kPa})(p<0.001)$ (Table 2).

However, only a weak correlation was found between size and stiffness measurements $(r=-0.32, p=0.1)$.

In the group of patients with metastases in the left liver lobe, a considerably higher increase in stiffness parameters was detected in the left lobe $(+72 \%, p=0.04)$ compared with the right lobe $(-37.5 \%, p>0.05)$ during the three cycles of TACE therapy. The left lobe parenchyma showed a significantly lower stiffness $(3.3 \pm 1.3 \mathrm{kPa})$ compared with the metastases $(5.4 \pm 1.8 \mathrm{kPa})$ (Fig. 4). The stiffness of the right lobe decreased from $4.0 \pm 1.6$ to $2.8 \pm 2.0 \mathrm{kPa}(p=0.1)$ during therapy (Table 3).

The stiffness of the liver as a whole slightly increased from $3.0 \pm 0.9 \mathrm{kPa}$ in the first cycle to $3.3 \pm 1.2 \mathrm{kPa}$ in the last cycle $(p>0.05)$. Measurements in the healthy liver tissue showed an increase in stiffness of only $+0.87 \%$ with an average of $2.2 \pm$ $0.6 \mathrm{kPa}$ during therapy $(p>0.05)$ (Table 4).
Table 2 Stiffness of different liver parts

\begin{tabular}{lcccccc}
\hline Measurement & Cycle 1 & Cycle 2 & Cycle 3 & Total & $p$ value & SD \\
\hline Area metastasis $\left(\mathrm{cm}^{2}\right)$ & 15.9 & 12.5 & 11.5 & 13.3 & 0.03 & 9.9 \\
Stiffness metastasis $(\mathrm{kPa})$ & 4.8 & 5.9 & 5.9 & 5.9 & $<0.001$ & 2.2 \\
Stiffness liver parenchyma $(\mathrm{kPa})$ & 3.0 & 3.2 & 3.3 & 3.2 & $>0.05$ & 1.0 \\
\hline
\end{tabular}


Fig. 4 TACE and MRE measurements in patients with colorectal liver carcinoma in the left liver lobe. A significant higher increase of stiffness $(+72 \%, p=0.04)$ was observed in the left lobe compared with the right lobe $(-37.5 \%, p>0.05)$ during the therapy. Additionally, the stiffness of the metastases was showing a distinct increase of stiffness $(52.4 \%, p=0.03)$

\section{TACE left lobe}

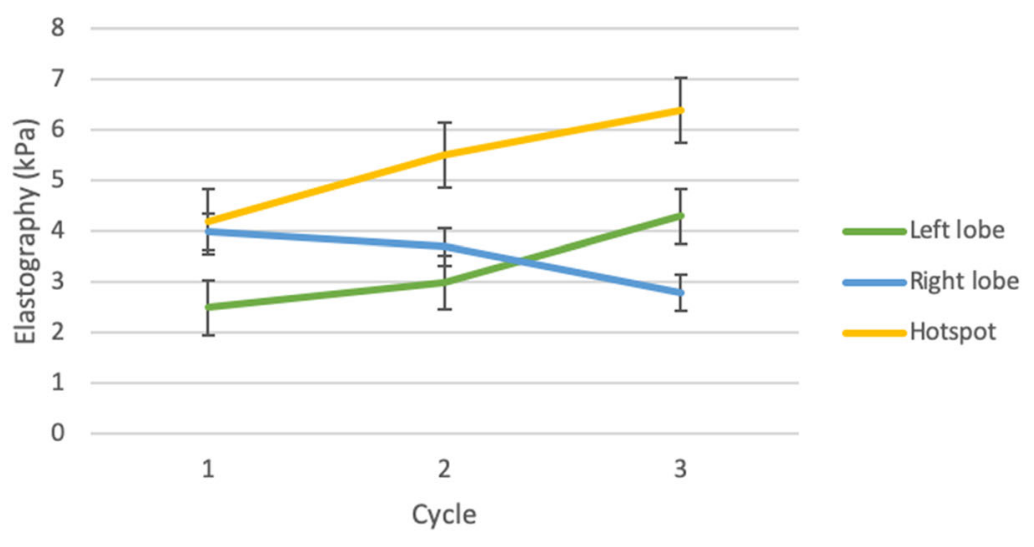

The MAP measurements showed fewer changes. The overall MAP T1 of the whole liver increased by $0.03 \%$ (from 636 \pm 226.7 to $655.2 \pm 236.4 \mathrm{~ms} ; p>0.05$ ) and the MAP T2 of the whole liver increased by only $0.003 \%$ (from $64.8 \pm 23.0$ up to $65.0 \pm 21.8 \mathrm{~ms} ; p>0.05)$. The MAP T1 and MAP T2 measurements of the metastases showed similar low changes than the rest of the liver. The MAP T2 in the metastases showed a significantly decrease (from $78.3 \pm 38.2$ to $76 \pm 38.1 \mathrm{~ms}$; $p=0.02)$ (Table 4).

\section{Discussion}

The aim of this study was to evaluate the therapeutic response of TACE in patients with colorectal liver metastases using MRI and MRE. We found that the additional use of elastography in the routine MRI protocol allows for the assessment of liver stiffness during interventional TACE cycles. MRE revealed significant differences in stiffness parameters for both normal liver lobes and metastases. This trend is due to the targeted accumulation of the drugs in the affected liver lobe without diffusion to the other lobe. Furthermore, a significant decrease in size and an increase in stiffness of the metastatic lesions demonstrated the beneficial therapeutic effects of TACE therapy. Although we have only found a weak correlation, the power of their validity should not be underestimated. For this reason, larger studies should be carried out to validate our results. The comparison of both liver lobes showed a lesser increase in stiffness in the untouched liver lobe compared with the treated lobe. Therefore, MRE can be used to evaluate the tumor response of colorectal liver metastases to chemotherapeutic substances. Furthermore, many patients undergoing TACE therapy have MRI or CT for staging and follow-up examinations, what insures a lot of data without additional imaging.

Gordic et al reported a different effect in their study in patients with hepatocellular carcinoma (HCC) who had undergone yttrium-90 radioembolization, TACE, or radiofrequency ablation (RFA) [23]. In their study, a significant lower stiffness was measured in treated tumors compared with untreated tumors. Even they showed a significantly higher accumulation of drugs in the tumor compared with the rest of the liver. A lower stiffness in HCC after therapy and a higher stiffness in CRLM after TACE might be explained by the different tumor entity. Moreover, the combination of different therapies in the study of Gordic et al compared with the single use of TACE in our study may have influenced the results. Venkatesh et al [24] investigated the stiffness of liver tumors with MRE. The authors demonstrated a non-significant linear correlation between the tumor size and stiffness. In contrast to our study, no homogeneous tumor was measured, but a large range of different lesions such as metastatic lesions (14, different primary tumor), hepatocellular carcinomas (12), hemangiomas (9), cholangiocarcinomas (5), focal nodular hyperplasia (3), and hepatic adenoma (1). Due to this big difference of tumor ethnicity, the validity of the results is not as powerful as in our
Table 3 Stiffness of liver at leftsided treated patients

\begin{tabular}{lllllrl}
\hline Measurement & Cycle 1 & Cycle 2 & Cycle 3 & Total & $p$ value & SD \\
\hline Area metastasis $(\mathrm{kPa})$ & 2.5 & 3.0 & 4.3 & 3.3 & 0.04 & 1.3 \\
Stiffness metastasis $(\mathrm{kPa})$ & 4.0 & 3.7 & 2.8 & 3.5 & $>0.05$ & 1.9 \\
Stiffness liver parenchyma $(\mathrm{kPa})$ & 4.2 & 5.5 & 6.4 & 5.4 & 0.03 & 1.8 \\
\hline
\end{tabular}


study with only one ethnicity. Moreover, the tumors were measured once and not in several phases. In another study, a correlation between tumor size and stiffness was also reported by Hennedige et al [25] as they demonstrated that benign and malignant lesions can be differentiated more significantly with MRE than with DWI.

Moreover, few studies evaluated the effects chemotherapy on liver stiffness in animal experiments. Pepin et al compared the stiffness of treated and untreated tumors in a mouse model [26]. After subcutaneous injection of non-Hodgkin's lymphoma cells in mice and subsequent treatment with a chemotherapeutic agent or saline, the authors detected a decrease of stiffness after 4 days in the tumors treated with chemotherapy and no appreciable change in the tumors treated with saline. Although there was no significant volume change detectable in this short time, the authors found a decreased level of cell proliferation [26]. In comparison with our study, neither the tumor entity nor the therapy was identical. Li et al demonstrated a similar decrease of stiffness in treated tumor cells compared with the non-treated cells in human colorectal cancer xenografts before and after treatment with vascular disrupting agent ZD6126 (N-acetylcolchinol-O-phosphate). The MRE took place $24 \mathrm{~h}$ after the treatment. In their analysis, a central necrosis was histologically confirmed [27].

These studies all demonstrate that MRE can be used for early response evaluation of tumor cells under therapy with chemotherapeutic agents. The studies showed in general a decrease of stiffness which is contrary to our results. This might be attributable to the different tumor origin and therapy in our study. Moreover, the accumulation of lipiodol in the metastases could have modified the stiffness of the lesions and may explain the different results compared with those in the literature.

Our results showed a higher degree of stiffness in metastases than in healthy liver areas measured. However, we found only a weak correlation between stiffness and size measurements of liver metastases. To our knowledge, no previous studies have analyzed the stiffness of colorectal liver metastases using MRI and MRE. Moreover, the difference between the left lobe and right liver lobe may be explained by the method, as the vibration source is located on the right anterior chest wall with intercostal approach. In this area, the compression might be less effective than in the left lobe which can be compressed by the elastic band that maintains the system. However, almost no drug-related effects were found in the healthy liver tissue.

MRE allows for highly accurate analysis of specific liver structures. Although ultrasound elastography has a higher plane resolution than MR, the depth is very limited [18]. The acquisition of EPI data took about 15 to $23 \mathrm{~s}$ for $1-5$ slices (WIP measurement), so a MRE measurement of the liver took on average up to $30 \mathrm{~min}$. Once the software is available, elastography measurements can be performed on every 1.5-T MR scanner with the use of the dedicated hardware and software packages.

There were certain limitations in our study. First, the patient cohort was relatively small, and we did not include a control group. Second, patients with different tumor stages were included in this study. The impact of TACE varies according to different tumor stages, as well as the measured stiffness. Third, patients with MRE were treated during different phases of TACE therapy. Some patients included in the current study received their first cycle while others had more than 20 therapies completed. Fourth, there were different periods of time between the start of therapy and image acquisition that may affect our data. As our study was in a retrospective setting and many patients received their therapy for a longer period of time, it would be interesting to evaluate MRE-based parameters in a prospective study with more patients. Furthermore, some of the MRE data were extrapolated that might also create bias [28]. In general, the quality of extrapolation is limited by the assumptions about the process of MRE values during therapy. Moreover, it remains unclear how accurate and repeatable MRE examinations are, even if the examination conditions are kept identically [28]. Therefore, studies with larger patient cohorts need to be conducted to confirm our results.

In conclusion, the MRI and MRE are useful tools to evaluate tumor response after TACE therapy in patients with colorectal liver metastases. Our results showed a significant increase in stiffness of the metastases and a simultaneous decrease in size of the metastases. Therefore, MRE imaging may provide additional value to evaluation of tumor response after TACE therapy. In addition, MRE measurements may be used
Table 4 Stiffness of different liver areas

\begin{tabular}{lcccccc}
\hline Measurement & Cycle 1 & Cycle 2 & Cycle 3 & Total & $p$ value & SD \\
\hline Healthy parenchyma & 2.2 & 2.2 & 2.2 & 2.2 & $>0.05$ & 0.6 \\
MAP T1 parenchyma (ms) & 636 & 678 & 655.2 & 656.4 & $>0.05$ & 217 \\
MAP T2 parenchyma (ms) & 64.8 & 70.4 & 65 & 66.7 & $>0.05$ & 21.4 \\
MAP T1 metastasis (ms) & 766.7 & 815.5 & 790.7 & 791 & $>0.05$ & 307.1 \\
MAP T2 metastasis (ms) & 78.3 & 86.2 & 76 & 80.2 & 0.02 & 37.1 \\
\hline
\end{tabular}


to calculate the remaining healthy liver proportion and function.

Funding Information Open Access funding provided by Projekt DEAL. The authors state that this work has not received any funding.

\section{Compliance with ethical standards}

Guarantor The scientific guarantor of this publication is Prof. Thomas J. Vogl.

Conflict of interest All authors of this manuscript declare no relationships with any companies whose products or services may be related to the subject matter of the article.

Statistics and biometry No complex statistical methods were necessary for this paper.

Informed consent Written informed consent was waived by the Institutional Review Board.

Ethical approval Institutional Review Board approval was obtained.

\author{
Methodology \\ - Retrospective \\ - Observational \\ - Performed at one institution
}

Open Access This article is licensed under a Creative Commons Attribution 4.0 International License, which permits use, sharing, adaptation, distribution and reproduction in any medium or format, as long as you give appropriate credit to the original author(s) and the source, provide a link to the Creative Commons licence, and indicate if changes were made. The images or other third party material in this article are included in the article's Creative Commons licence, unless indicated otherwise in a credit line to the material. If material is not included in the article's Creative Commons licence and your intended use is not permitted by statutory regulation or exceeds the permitted use, you will need to obtain permission directly from the copyright holder. To view a copy of this licence, visit http://creativecommons.org/licenses/by/4.0/.

\section{References}

1. Torre LA, Bray F, Siegel RL, Ferlay J, Lortet-Tieulent J, Jemal A (2015) Global cancer statistics. CA Cancer J Clin. https://doi.org/ 10.3322/caac. 21262

2. Ihnát P, Vávra P, Zonca P (2015) Treatment strategies for colorectal carcinoma with synchronous liver metastases: which way to go? World J Gastroentrerol. https://doi.org/10.3748/wjg.v21.i22.7014

3. McMillan DC, McArdle CS (2007) Epidemiology of colorectal liver metastases. Surg Oncol. https://doi.org/10.1016/j.suronc. 2007.04.008

4. Van Cutsem E, Köhne CH, Hitre E et al (2009) Cetuximab and chemotherapy as initial treatment for metastatic colorectal cancer. N Engl J Med. https://doi.org/10.1056/NEJMoa0805019

5. Simmonds PC, Primrose JN, Colquitt JL, Poston GJ, Rees M (2006) Surgical resection of hepatic metastases from colorectal cancer: a systematic review of published studies. Br J Cancer. https:// doi.org/10.1038/sj.bjc.6603033
6. Scheele J, Stangl R, Altendorf-Hofmann A (1990) Hepatic metastases from colorectal carcinoma: impact of surgical resection on the natural history. Br J Surg 77(11):1241-1246

7. Bruix J, Castells A, Bosch J et al (1996) Surgical resection of hepatocellular carcinoma in cirrhotic patients: prognostic value of preoperative portal pressure. Gastroenterology 111(4):1018-1022

8. Cohen AD, Kemeny NE (2003) An update on hepatic arterial infusion chemotherapy for colorectal cancer. Oncologist. https://doi. org/10.1634/theoncologist.8-6-533

9. Bentrem DJ, Dematteo RP, Blumgart LH (2005) Surgical therapy for metastatic disease to the liver. Annu Rev Med 56:139-156

10. Ji SH, Park YS, Lee J et al (2005) Phase II study of irinotecan, 5fluorouracil and leucovorin as first-line therapy for advanced colorectal cancer. Jpn J Clin Oncol. https://doi.org/10.1093/jjco/hyi061

11. Kuehr T, Ruff P, Rapoport BL et al (2004) Phase I/II study of firstline irinotecan combined with 5-fluorouracil and folinic acid Mayo Clinic schedule in patients with advanced colorectal cancer. BMC Cancer. https://doi.org/10.1186/1471-2407-4-36

12. Kemeny N, Garay CA, Gurtler J et al (2004) Randomized multicenter phase II trial of bolus plus infusional fluorouracil/leucovorin compared with fluorouracil/leucovorin plus oxaliplatin as third-line treatment of patients with advanced colorectal cancer. J Clin Oncol 22(23):4753-4761

13. Vogl TJ, Zangos S, Eichler K, Yakoub D, Nabil M (2007) Colorectal liver metastases: regional chemotherapy via transarterial chemoembolization (TACE) and hepatic chemoperfusion: an update. Eur Radiol. https://doi.org/10.1007/s00330-006-0372-5

14. Germer CT, Buhr HJ, Isbert C (2005) Nonoperative ablation for liver metastases. Possibilities and limitations as a curative treatment. Chirurg 76(6):552-554 556-63

15. Bavisotto LM, Patel NH, Althaus SJ et al (1999) Hepatic transcatheter arterial chemoembolization alternating with systemic protracted continuous infusion 5-fluorouracil for gastrointestinal malignancies metastatic to liver: a phase II trial of the Puget Sound Oncology Consortium (PSOC 1104). Clin Cancer Res 5(1):95-109

16. Vogl TJ, Reith W, Rummeny EJ (2011) Diagnostische und Interventionelle Radiologie, 1st edn. Springer-Verlag, Berlin, Heidelberg

17. Wallace $\mathrm{S}$, Carrasco $\mathrm{CH}$, Charnsangavek C, Richli WR, Wright $\mathrm{K}$, Gianturco C (1990) Hepatic artery infusion and chemoembolization in the management of liver metastases. Cardiovasc Intervent Radiol 13(3):153-160

18. Vogl TJ, Mack MG, Balzer JO et al (2003) Liver metastases: neoadjuvant downsizing with transarterial chemoembolization before laser-induced thermotherapy. Radiology. https://doi.org/10.1148/ radiol.2292021329

19. Legou F, Chiaradio M, Baranes L et al (2014) Imaging strategies before beginning treatment of colorectal liver metastases. Diagn Interv Imaging. https://doi.org/10.1016/j.diii.2014.03.007

20. Venkatesh SK, Ehmann RL (2014) Magnetic resonance elastography of liver. Magn Reson Imaging Clin N Am. https:// doi.org/10.1016/j.mric.2014.05.001

21. Gennisson JL, Deffieux T, Fink M, Tanter M (2013) Ultrasound elastography: principles and techniques. Diagn Interv Imaging. https://doi.org/10.1016/j.diii.2013.01.022

22. Vogl TJ, Schaefer P, Lehnert T et al (2016) Intraprocedural blood volume measurement using $\mathrm{C}$-arm $\mathrm{CT}$ as predictor for treatment response of malignant liver tumors undergoing repetitive transarterial chemoembolisation (TACE). Eur Radiol. https://doi. org/10.1007/s00330-015-3869-y

23. Gordic S, Ayache JB, Kennedy P et al (2017) Value of tumor stiffness measured with MR elastography for assessment of response of hepatocellular carcinoma to locoregional therapy. Abdom Radiol (NY). https://doi.org/10.1007/s00261-017-1066-y 
24. Venkatesh SK, Yin M, Glockner JF et al (2008) MR elastography of liver tumors: preliminary results. AJR Am J Roentgenol. https://doi. org/10.2214/AJR.07.3123

25. Hennedige TP, Hallinan JT, Leung FP et al (2016) Comparison of magnetic resonance elastography and diffusion-weighted imaging for differentiating benign and malignant liver lesions. Eur Radiol. https://doi.org/10.1007/s00330-015-3835-8

26. Pepin KM, Chen J, Glaser KJ et al (2014) MR elastography derived shear stiffness - a new imaging biomarker for the assessment of early tumor response to chemotherapy. Magn Reson Med. https:// doi.org/10.1002/mrm.24825
27. Li J, Jamin Y, Boult JK et al (2014) Tumor biomechanical response to the vascular disrupting agent ZD6126 in vivo assessed by magnetic resonance elastography. Br J Cancer. https://doi.org/10.1038/ bjc. 2014.76

28. Serai SD, Obuchowski NA, Venkatesh SK et al (2017) Repeatability of MR elastography of liver: a meta-analysis. Radiology. https://doi.org/10.1148/radiol.2017161398

Publisher's note Springer Nature remains neutral with regard to jurisdictional claims in published maps and institutional affiliations. 